\title{
EL GATO MACHI Epew pewenche
}

\author{
Gilberto Sánchez Cabezas* \\ Universidad de Chile, Chile
}

\begin{abstract}
El siguiente epew nos fue narrado por Lorenzo Naupa Epunán, un kimche ${ }^{1}$ pehuenche, cuya temprana desaparición no terminamos de lamentar. Era un profundo conocedor de su cultura y un narrador excepcional, cuyo discurso podía durar horas en chedungun (pehuenche del Alto Bío-Bío), con muy pocas palabras del wingkadungun (español) intercaladas. El epew es como una novela breve y narra el contrapunto entre el león y su incorregible sobrino, el zorro. Este, que suele "pensar mal", no termina bien. Su mal comportamiento es una advertencia para quienes escuchan el relato. El escenario es la cordillera. Una vez, cuando llega el "año nuevo" -el 24 de junio- la necesidad (villa), es decir, la falta de víveres, afecta a un matrimonio de leones. Los pehuenches suelen recordar el hambre que sufrían las comunidades, sobre todo durante el riguroso invierno - con copiosas lluvias y nevazones-, cuando se dependía mayormente para sobrevivir de los frutos de los pinos $\left(\right.$ pewen $\left.^{2}\right)$. Por la necesidad el león decide viajar lejos a buscar 'mantención' (mongewe), a la Tierra del Oriente (Argentina). Antes los
\end{abstract}

Para correspondencia, dirigirse a: Gilberto Sánchez Cabezas (gsanchez@uchile.cl), Universidad de Chile, Facultad de Filosofía y Humanidades, Departamento de Lingüística, Av. Capitán Ignacio Carrera Pinto 1025, Ñuñoa, Santiago, Chile.

1 Persona entendida, conocedora, sabia. Se dice también kimwentru. L. Naupa falleció trágicamente en octubre de 1981. El epew lo narró el 23 de marzo ese mismo año. Nos entregó otros textos orales, algunos de los cuales ya hemos publicado.

2 El pino araucaria (Araucaria araucana). 
pehuenches acostumbraban ir allá a proveerse de los "vicios" (yerba mate, azúcar, etc.), pero también a vender y "conchabar" (intercambiar) sus propios productos (tejidos, yerbas medicinales). El viaje tenía algunos riesgos -por eso el zorro lleva cuchillo y revólver-, pero valía la pena realizarlo. En este caso, el león trajo abundante ganado desde la Tierra del Oriente. Los conflictos con el zorro - debido a que solía andar con flojera- ocasionan que su tía -la esposa del león- lo castigue al regresar. Entonces anuncia que, como se ha sentido muy enfermo, se va a morir; también porque su abuela paterna $(k u k u)$ lo ha venido a ver desde "la tierra de arriba" (el wenumapu). El león no le cree, pero el zorro efectivamente muere. En vista de ello -y seguramente por remordimiento- el león decide buscar a un $m a c h i^{3}$, para que lo haga resucitar, el cual resultó ser un gato, llamado Hualquipayún. Se cumplen las condiciones que impone el machi para efectuar el acto de sanación. En la ceremonia participan varios pájaros, y hasta un sapo, con ejecución de toques de kultrún ${ }^{4}$ y gritos de kavaván ${ }^{5}$. También tiene lugar la instalación del árbol sagrado del machi (ñaykurewen) El gato - que conocía muy bien la astucia del zorro- sospecha que la muerte es fingida y, por eso, decide al cabo aplicarle, varias veces, una cala de hormigas coloradas en el ano, cuya picada no puede soportar mucho rato y, de inmediato, resucita y huye dando grandes saltos. Por ello es objeto de burlas, sobre todo de parte de la bandurria, la cual se ríe a carcajadas de él. El epew concluye aseverando que, desde entonces, existe una enemistad entre el gato y el zorro y que este, apenas ve al primero, de inmediato lo persigue.

La lengua del texto es la variedad pewenche (chedungun) del mapudungu(n), hablada en la comunidad de Cauñicú, en el Alto BíoBío. Fonológicamente se caracteriza por la ocurrencia de los fonemas /v/ labiodental fricativo oral sonoro y /d/ interdental fricativo oral áfono, los cuales no ocurren en otras variedades del mapudungu(n). Es un rasgo fonológico conservador. Morfológicamente no presente diferencias marcadas respecto de otras variedades regionales. El chedungun resulta inteligible para hablantes de otros sectores del territorio mapuche. Respecto del léxico, posee muchas palabras para referirse al hábitat cordillerano y al pewen (pino Araucaria araucana).

Curandero/a mapuche.

Tambor empleado por el (la) machi en ceremonias de curación y de ngillatun.

Gritos intermitentes proferidos para alejar a los malos espíritus. 
La grabación -de 50 minutos de duración- ha sido transcrita empleando el Alfabeto Mapuche Unificado ${ }^{6}$, a fin de hacer más accesible el texto a estudiosos de la etnoliteratura que no son lingüistas. Solo se representan en él las pausas que ocurren entre cada macrosegmento y la entonación. [l] indica pausa mínima (menos de tres segundos); [ |l] , pausa normal (de tres a cinco segundos] y [III], larga o morosa (más de cinco segundos). Las inflexiones terminales se representan con $[\rightarrow]$, tensa sostenida; [ $\uparrow]$, ascendente y $[\downarrow]$ descendente. En el relato predomina la inflexión descendente. Cuando en un macrosegmento su centro es muy prominente, se representa con ['].

Dado que se trata de un texto oral, los ilativos veymu, vey, vey ta, veymu vey, vey veymu ${ }^{7}$, vey, y sus combinaciones veymu vemngechilu, vey ta, vey vemngechilu vey, tienen una alta frecuencia. Ellos cumplen la función de unir los macrosegmentos y mantener la atención de quienes escuchan el relato (en este caso, fueron los miembros del grupo familiar ${ }^{8}$ ). No siempre los hemos traducido, para no recargar demasiado la traducción en español. No hemos traducido el morfema reportativo $\{-r k e-\}$ y, solo parcialmente, el impersonal piam, los cuales significan 'dicen que'. Al emplearlos el narrador indica que los sucesos referidos en el epew no los ha presenciado, sino que los ha oído de otra(s) persona(s). Ambos tienen también una alta frecuencia.

La traducción que se entrega es semilibre, es decir, no es ni literal ni se aparta demasiado del original (para no traicionarlo). No pretende ser perfecta, pues es una tarea difícil traducir fielmente de una lengua que posee una estructura completamente diferente de la del español y representa, además, una cultura muy sui géneris.

El texto, además de su valor como tal, da testimonio de una práctica cultural ya desaparecida en el Alto Bío-Bío, desde hace mucho tiempo: el machitun. Antes habría existido, pero los pehuenches actuales no pueden precisar cuándo dejó de realizarse, tampoco por cuales motivos. El narrador no explicó en qué consistía el ñaykurewen.

Para informarse acerca del procedimiento de recolección en terreno, características formales de los relatos, etc., remitimos a nuestras publicaciones.

${ }^{6}$ Solo hemos sustituido el grafema $f$ por $v$, porque el chedungun de Cauñicú no posee el fonema $/ \mathrm{f} /$.

7 Veymu puede traducirse por 'entonces', 'luego', por eso', 'con eso', 'ahí'; vey, por 'entonces', 'luego', 'en este o ese momento', 'y'.

8 Disfrutaron con el epew y, en más de un pasaje, se rieron de buena gana; también el narrador. 
1. veymu tüvachi travia $\rightarrow \mid$ ká püchin 1 . Entonces, esta noche le contaré otro ta epewelaviñ ta tachi utrangepalu ${ }^{9} \rightarrow \|$ poco de epew a esta visita que ha venido aquí...

2. kuyvi mu $\rightarrow$ eypi ngütram pütake 2 . Antiguamente conversaban nuestros che em $\rightarrow \mid$ mülekerki kiñe kurewen finados padres que había un matrimonio püchapeñ $i^{10} \downarrow \mid$ kiñe mapu mu de leones. Vivían en un campo (y) tenían mülekerki $\rightarrow \mid$ nierki kiñe ruka $\downarrow \mid$ epu una casa. Eran solo dos. Tenían también mülekerki müten $\downarrow \mid$ vey ka kiñe werken ${ }^{11}$ un mozo llamado zorro.

niekerki $\rightarrow \mid$ ngürü $^{12}$ pingi $\downarrow \mid$

3. veymu kiñerupa $\uparrow \|$ wetripantiñmaalu $\rightarrow \mid 3$. Entonces, una vez, al llegar el año villarki tati püchapeñi $\downarrow \mid$ veymu dewma nuevo ${ }^{13}$, sufrió escasez el león. Cuando ya villalu $\uparrow$ vey ngütramkawürki kurewen $\downarrow \mid$ sufría escasez, conversó el matrimonio.

4. kiñe pañü eypirki püchapeñi ellawün $\downarrow$ 4. Un día dijo el león, temprano: “-Ahora “-tüva nga wetripantiñmayñ dewma nga ya estamos en el año nuevo y nos está dilepay nga villa ${ }^{14}$ " $\downarrow \mid$ pirki tayñi ngütram alcanzando la escasez”, conversó el león, püchapeñi $\rightarrow$ eypirki ñi kure| llamngen le dijo a su mujer, que se llamaba leona. pingi $\downarrow$

5. “-veynollanchi个”15 pirkitallamngen $\downarrow$ "- 5. “¡Así es, pues!”, dijo la leona. ñal nga avüle nga mongewe $\rightarrow \mid$ "chew "-Cuando se nos acabe la mantención, nga peayu nga tüva $\uparrow$ | “-pütrüchipali ¿dónde encontraremos?” “-Está harto nga ye-men” pirki llamngen $\downarrow$ lejos para ir a buscar", dijo la leona.

6. “vey anta rakiduampellelan $\downarrow ” \mid$ pirki 6. “-¡Eso es lo que pienso!”, dijo el león. pü-chapeñi $\downarrow \mid$ “"-veymu rakiduampen “-jPor eso estoy pensando, pues!”, dijo tata” $\rightarrow$ pirki tayñi kure ta püchapeñi $\downarrow$ el león a su mujer. “-Estoy decidido “-nampülkan pipen tata $\downarrow$ amon a viajar lejos. Voy a ir al Tierra del

\footnotetext{
9 GSC.

10 Nombre que se da al león (Felis concolor) en chedungun y significa 'hermano mayor'; la leona es llamada pücha llamngen 'hermanita mayor', o simplemente llamngen 'hermanita'.

11 El narrador tradujo werken por 'mozo'. La palabra ha significado siempre 'mensajero' y 'mensaje'.

12 Aunque no se especifica, debe tratarse del zorro kulpew (Lycalopex culpaeus, Pseudalopex culpaeus).

13 El wetripantu el año nuevo mapuche, comienza el 24 de junio.

14 "Villa - carestía, hambruna, esterilidad de víveres, y mantenimiento, etc. (Febrés, Calepino: 661).

15 Literalmente ‘¿no es así, pues?’; equivale a una afirmación.
} 
puelmapu"16 $\rightarrow$ pirki tayñi ngütram Oriente (Argentina)", dijo el león (en su püchapeñi $\downarrow$ conversación).

7. "vente tripaamoymi tiye püta wentu" 7. "-Muy lejos vas a salir viejito", le dijo pirkeyu tayñi llamngen $\downarrow$ “-eyew amon su leona. “-¡Iré allá, pues! No habiendo nga $\downarrow$ kaypüle ngenolu ngay $\rightarrow \mid$ chew en otra parte, alcanzaré hasta donde mülemom diton" pirki ta püchapeñi $\downarrow$ haya", dijo el león. “-Tú sabrás”, se le vey "-kidu ngü- neymi"" $\rightarrow \mid$ pingerki dijo, le dijo la leona.

eypieyu llam-ngen $\downarrow$

8. veymu ngütrümngerki ngürü $\downarrow \mid$

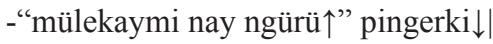

8. Entonces llamaron al zorro.

-“chem” pirki ngürü $\downarrow$

“-¿Estás todavía ahí zorro, pues?”, le

“künapange" $\downarrow$ pirkeyu ta püchapeñi $\downarrow \mid$ “-Ven”, le dijo el león. "-Te necesito, “-du-amyepeyu anü malle" pingerki $\downarrow$ amigo sobrino ${ }^{18} "$, le dijo.

“-veyllepu malle $\downarrow \mid$ müli tam duam nga” $\downarrow$ “-Está bien, tío. Me necesitas, pues”, le pingerki püchapeñi $\downarrow$

dijo al león.

9. “-rakiduampen tata vey püchi 9. "-Estoy pensando, quiero viajar un nampülkan pipen anü malle $\downarrow$ poco lejos, amigo sobrino. ¿Cómo te chumkülevuymi diweñaven anü” $\uparrow$ hallarías para acompañarme, amigo?”, “-amon puelmapu pipen tata” $\downarrow$ pirki ta dijo el león. "Quiero ir a la Tierra del püchapeñi $\downarrow$

Oriente", dijo el león.

10. vey $\uparrow$ ayenagi ñi nge ngürü $\downarrow \mid$

10. Entonces se le pasó la alegría ${ }^{20}$ al “-vey nollanchi nay malle $\uparrow "$ pirki ta zorro.

ngürü $\downarrow \mid$ “-kawellu müten püchi küntroli “-¡Como no, pues, tío!”, dijo el zorro. anü” pirki ngürü $\downarrow$

-“no eypikelmi $\downarrow$ inche altrolayu kiñe küme kollu" ${ }^{19}$ " pingerki $\downarrow$

"-(Mi) caballo nomás está un poco cojo",

-"vey vemngechi altroveli kawellu vey

ta di-weñkaveyu anü malle" $\rightarrow$ pirki ta ngürü $\downarrow$

dijo el zorro.

"-No digas eso. Yo te voy a pasar un buen caballo", le dijo.

“-Así, si me pasaras un caballo, te acompañaría, amigo tío”, dijo el zorro.

16 “Oriente -puel ple, ò mapu” (Febrés, Calepino: 378), "puelmapu, s.c., la Argentina” (Augusta, Diccionario I: 188).

17 Literalmente 'tu te mandas solo'.

18 El león es tío paterno (malle) del zorro. El término es recíproco y, por consiguiente, significa también 'sobrino paterno'.

19 Palabra usual en Cauñicú, en vez de kawellu.

20 Literalmente 'dejaron de reírsele los ojos'. No le gustó la invitación. 
11. “-ya $\uparrow$ amoyu anü $\downarrow \mid$ eppwe pürakolloyu” pingerki $\downarrow$ |"-tay kintukollukünoayu vey ule kümeluayu kom livtukawayu takuñ rokiñwayu vey eppue amoyu $\downarrow$ püchi upale travia chillakolloyu amoyu $\downarrow ” \mid$ pirki püchapeñi $\downarrow \mid$

12. vey $\uparrow \mid$ "-yavuluan $\downarrow$ ” vey küchatumen pirki ta ngürü $\downarrow$ “-küchamen makuñ anü” pirki $\downarrow$ |“-nien kiñe deya eyew vey küchavalmen” pirki $\downarrow$

13. vey vemngechirkingu $\downarrow \mid$ vey ká wünpüle kom angkümlu makuñ engu kom rokiñwü--rkingu $\rightarrow \mid$ veymu eypirki püchapeñi $\downarrow$

-"kiñe epu antü rüpütun rokiñ ${ }^{22}$ küüdawelan $\downarrow " \mid$ pingerki ta llamngen $\downarrow \mid$ -“ya $\uparrow "$ pirki llamngen $\downarrow$ kümelelngerki epu antü tayñi awantaalu tati iagel tayñi rokiñ $\downarrow$

14. veymu| epu entu mu (pü) chi upay travia amurkingu $\downarrow \mid$ kiñetu ngütrüm achawall mu vey ta chillakolluy püchapeñi vey malle kavey ngürü $\downarrow \mid$

15. -“itrokom yeymi nay^"| "-yeymi llasu ka küme kuchillúu $\uparrow$ pingerki ta ngürü $\downarrow \mid$

-“may itrokom trayintukuneuyen” pirki $\downarrow$

“-küme llasu küme kuchillu ka kiñe regol-vé $\downarrow$ "

"-vey ta chay tiepüle katrütungeliyu" $\rightarrow \mid$ pirki ta püchapeñi $\downarrow \mid$
11. “-Bueno, vamos, amigo. Pasado mañana cabalgaremos", le dijo. "Hoy buscaremos caballos, mañana nos alistaremos, limpiaremos toda la ropa, prepararemos el cocaví, y pasado mañana nos iremos. Cuando pase un poco la noche ensillaremos (y) nos iremos", dijo el león.

12. "-Le voy a hacer empeño, voy a ir a lavar dijo el zorro". "-Voy a ir a lavar la manta", dijo. "-Tengo una hermana ${ }^{21}$ allá, le voy a mandar a lavar”, dijo.

13. Así lo hicieron. Luego, al otro día, cuando secaron las mantas (y) prepararon todo el cocaví, entonces dijo el león:

“-Prepárame cocaví para uno o dos días de viaje". (Eso) le dijo a la leona. “-¡Está bien!", dijo la leona.

Se le preparó cocaví, comida, para que le aguantara dos días.

14. Entonces, al segundo día, un poquito pasada la media noche, se fueron los dos. Con el canto del gallo, ensilló el león y también su sobrino, el zorro.

15. “-¿Llevas todito, pues?”. “LLlevas lazo y un buen cuchillo?", le dijo al zorro.

"-Sí, todito lo llevo ya amarrado", dijo, "-un buen lazo, un buen cuchillo y un revólver".

"-Por si luego nos asaltan por ahí", dijo el león.

21 Deya puede significar también 'prima de un hombre'.

22 "rokiñ, s., la provisión para el viaje" (Augusta, Diccionario I: 200). La palabra se emplea también al hablar en español. 
16. vey pürakollurkingu $\downarrow$

16. Montaron a caballo los dos.

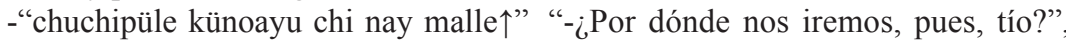
pirki ta ngürü $\downarrow$ dijo el zorro.

-“-no tüva mu wivod mu amoyu $\downarrow$ ” pirki “-Bueno, aquí por el filo de montaña nos ta püchapeñi $\downarrow \mid$ iremos", dijo el león.

17. püt-ta wivod mu amuy $\downarrow \mid$ amurkingu 17 . Se fueron por un filo grande pü-rayngu kom antü rüpüturkingu $\downarrow$ de montaña. Caminaron, subieron, traviayñ- mapoyngu angkaporkingu caminaron todo el día. Les anocheció. wivod mu $\rightarrow$ vey umañkünoporkingu $\downarrow$ Llegaron hasta la mitad del filo de la umañpoyngu $\downarrow$ vey nielay che piam montaña. Entonces se dispusieron a püt-takamapu piam tati puelmapu $\downarrow$ ni dormir. Alojaron allá. Dicen que no ume ngelay piam nga veychipüle $\downarrow$ hay gente, está muy lejos la Tierra del Oriente. No hay nadie por ahí.

18. veymu $\uparrow$ ká epe wünmaalu engu $\rightarrow$ vey ey pirki ta püchapeñi $\downarrow$ "-ranginpay weluwitraw ${ }^{23}$ anü malle $\downarrow " \mid$ "-ká amoyu” pirki püchapeñi $\downarrow$ vey ká chillakolluingu $\rightarrow$ ká pürakolluingu $\rightarrow$ ká amuingu $\rightarrow \|$ ká amuingu ká kiñe pañü amurkingu $\downarrow \mid$

18. Entonces, casi al amanecerles, dijo el león: "-Llegaron a la mitad (del cielo) "Las Tres Marías", amigo sobrino". “-Sigamos caminando", dijo el león. De nuevo ensillaron, montaron a caballo y se fueron. Se fueron, caminaron otro día más.

19. veymu karalka ${ }^{24}$ chovüy piam $\rightarrow$ ka- 19 . Entonces le dio flojera al zorro. No se ralka $\mid$ ngürü $\downarrow \|$ pep-pulay piam kiñe che veía a nadie. Dicen que no hay gente en ume $\downarrow \mid$ nielay piam che tiechi mapu mu| esa tierra, en esa tierra grande solitaria. püt-ta kidulechi mapu mu $\downarrow$ re mawida Dicen que es pura montaña. piam $\downarrow$

20. veymu $\uparrow$ ká traviaymapoy piam $\downarrow \mid$ vey 20 . Entonces, otra vez les anocheció (y) avpoy piam rokiñ engu $\downarrow$ se les acabó el cocaví.

“-na weda anay malle| avuy rokiñ anü $\downarrow " \mid$ “-Muy malo está, pues, sobrino. Se acabó pirki püchapeñi $\downarrow$

“-avuy nay malle" pirki $\downarrow$ el cocaví, amigo”, dijo el león. “-Se acabó, pues, tío", dijo.

"-chem am iayu tüva $\uparrow$ nielay tüvapüle "-¿Qué vamos a comer ahora? No hay che chuchi mu püchi ngillatom" pirki por aquí gente a quien pedir un poco", pü-chapeñi $\downarrow$ dijo el león.

niküvuli piam karalka ngürü $\downarrow \mid$ püchi Dicen que se quedó callado el zorro. weya-duamüy piam ta ngürü $\downarrow \mid$ ngüñelu Pensó mal un poco el zorro ${ }^{25}$. Tenía piam $\downarrow$ hambre. de Orión.

23 "Las Tres Marías" (Alnitak, Alnilam y Mintake). Forman el cinturón de la constelación

24 Otro nombre para el zorro, en chedungun.

25 El zorro suele pensar mal, tener malas intenciones. 
21. veymu wünpüle ká epewün ká 21. Entonces, al alba del día siguiente, chillakolluingu piam $\downarrow$ doy rangiñrüpü- volvieron a ensillar. Llegaron a más de la poingu piam $\downarrow \mid$ ká amukünowingu tripa- mitad del camino. De nuevo se pusieron upoyngu kiñe püt-ta wivod $\mathrm{mu} \rightarrow \mid$ en camino (y) y salieron por un gran filo nagkintupoyngu piam kiñe püt-ta lom de montaña. Dicen que miraron hacia un piam $\downarrow \mid$ gran bajo.

22. veymu mülerki kiñe ülmen ${ }^{26} \rightarrow \mid$ nierki 22 . Ahí vivía un caballero rico. Tenía kullin nierki ovisa kapüra nierki ká animales: ovejas, cabras; también tenía nierki waka kawellu nierki ta yewüntu $\rightarrow$ vacas, caballos. Tenía aves: gallinas, achawall alkapio kolkol kom nierki $\downarrow$ gallos, pavos. Tenía de todas.

23. malle piam ngürü ta inali piam $\uparrow \| 23$. El sobrino zorro iba detrás. Iba con chovün amuy piam $\downarrow \mid$ veymu püchi flojera. Entonces el león esperó un poco üngelpoy piam ñi $\operatorname{moso}^{27}$ ta püchapeñi $\downarrow$ a su mozo. Al no llegar pronto allá el awediponolu ngü- trümngi piam ta zorro, fue llamado:

ngürü $\rightarrow \mid$

“-chew anta küpalimi nay malle $\uparrow$ pipurki ta püchapeñi $\downarrow$

“-¿Dónde venías, pues, sobrino?”, le dungulay piam ngürü ka lladküy piam enojó. Tenía hambre.

ngürü $\downarrow \mid$ ngüñelu $\downarrow \mid$

24. "-chew anta küpalimi nay malle $\uparrow " \mid$ pirkeyu ula püchapeñi| lladküy püchapeñi $\downarrow$

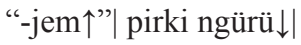

“-matukelpange anay $\uparrow ”$ pingerki $\downarrow$ amuy piam ngürü $\downarrow$

dijo el león.

Dicen que no habló el zorro y que se “-tüva müli kiñe ülmen anü malle tüva mu püchi ngillatoyu ial” pingerki 24. “-¿Dónde venías, pues, sobrino?”, le dijo luego el león; se enojó el león.

“-¡Qué puh!”, dijo el zorro.

“-¡Apúrate pues!”, le dijo.

Dicen que fue el zorro.

"-Aquí vive un caballero rico, amigo ngürü $\downarrow \|$

25. nagkolluy piam ngürü| minche virken anükünowi $\downarrow \mid$ vey püchapeñi ngünewlay ñi nagal| ngünewlay ñi konpual ruka $\mathrm{mu} \downarrow$ sobrino. Aquí vamos a pedir un poco de comida", le dijo al zorro.

25. Se bajó del caballo el zorro (y) se sentó a la sombra. El león no se animó a bajarse del caballo, no se animó a entrar en la casa.

26 Ülmen: cacique, persona rica. "Ghùlmen - Cazique, y hombre rico, y de respeto" (Febrés, Calepino: 490).

27 El narrador empleó la palabra española, en vez de werken. Véase nota n. ${ }^{\circ} 10$. 
26. veymu eypingerki ngürü $\downarrow$

"-eymi yavulunge anü malle $\uparrow$ entumeymi

kiñe ovisa| kangkatoyu anü" pingerki ta ngürü $\downarrow$

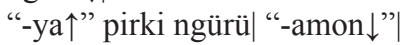

“-tapüle weletringkaykünomeaymi”| pinge- rki ta ngürü pirkeyu püchapeñi $\downarrow \mid$

27. nagi piam ngürü $\downarrow \mid$ pukepuke konpoy piam ngürü lemu mu $\downarrow$ püchi llikali piam ñi amun $\downarrow \mid$ veymu kiñe lemu mu konpoy ngürü $\downarrow$

28. veymu mülerki kiñe kurewen| alkapio ka kiñe kuse achawall engu $\downarrow$ veymu ti kuse achawall perkeyu| alkapio perkelayu $\downarrow$

“-chumpi anay ti akuchachi wün $\uparrow \mid$ pirki

ti kuse achawall $\downarrow$

wiñokintuvemüy piam ta alkapio $\downarrow \mid$ veymu eypirki ta alkapio $\downarrow$

“-püt-ta pültrükütraw $\uparrow " 29$ pingerki ngürü $\downarrow$

lladküy piam ngürü $\downarrow$

“-akuchachi wün $\uparrow$ ká pikarkeyu ti kuse

achawall $\downarrow$ ká pingekarki ngürü $\downarrow$

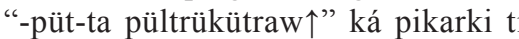

alka-pio $\downarrow$.

29. veymu $\rightarrow \mid$ “-pültrükütraw pien nay $\uparrow ” \mid$ pingerki alkapio $\downarrow \mid$ veymu vey ta eypirki ñi rakiduam ngürü $\downarrow \mid$ “"-tüvangechi yeeviñ anü” pirki $\downarrow \mid$ “-to kangkatoviñ ” pirki $\downarrow$ eypi raki- duamürkevi ti alkapio $\downarrow \|$

30. vey ñochikechi kontuki piam iñetripaki piam ti alkapio $\downarrow$
26. Entonces le dijo al zorro:

“-Hazle empeño tú, amigo sobrino; anda a sacar (traer) una oveja. Haremos asado, amigo", le dijo al zorro.

“-¡Está bien!”, dijo el zorro. “-Voy”.

“-Por aquí, por el lado izquierdo vas a ir a rodearla" 28 , le dijo el león al zorro.

27. Bajó el zorro. Con recelo entró el zorro en el bosque. Dicen que iba con un poco de miedo. Entonces entró en un bosque el zorro.

28. Ahí vivía un matrimonio, un gallo y una gallina vieja. Entonces lo vio la gallina vieja; el gallo no lo vio.

"-¿Qué anda haciendo, pues, el hocico de aguja?", dijo la gallina vieja.

Miró de repente hacia atrás el gallo. Entonces dijo el gallo:

"-¡Testículos grandes que (te) cuelgan!", le dijo al zorro. Dicen que se enojó el zorro.

“-¡Hocico de aguja!”, volvió a decirle la gallina vieja al zorro.

“-¡Testículos grandes que (te) cuelgan!", i volvió a decir el gallo.

29. Luego: “-¡Me dijiste testículos que (te) cuelgan, pues!", le dijo al gallo. Entonces pensó el zorro: “-A este me lo voy a llevar, pues, lo voy a hacer asado". Dicen que eso pensó el zorro del gallo.

30. Se acercaba despacito, (pero) el gallo se retiraba.

28 Para lograrlo tendría que haber entrado por el lado derecho, de la buena suerte.

29 Pültrün: colgar, estar colgado. "kütrau, s., el testículo, compañón” (Augusta, Diccionario I: 107). 
vey püchapeñi piam kintuleki $\rightarrow \mid$ chem El león estaba (entretanto) mirando. weda ngüñeli piam $\downarrow$ Dicen que tenía muchísima hambre.

veymu piam ngürü pepi nükelavi Entonces el zorro no podía agarrar al ti alkapio $\downarrow \mid$ trilal-leki piam ngürü $\uparrow \mid$ gallo.

iñekontuki piam ti achawall $\downarrow \mid$ vemmeki ¡Estaba tirado de espaldas el zorro! La piam $\uparrow \mid$ gallina volvía a acercarse. ¡Dicen que en eso estaban!

31. veymu t'üm na lladküy piam pücha- 31. Entonces se enojó muchísimo el león. peñi $\downarrow$ “"-werkeviñ nga ti ngamno ${ }^{30}$ ta “-Lo mandé al bribón, y anda tüngkeliawtuy” $\downarrow$ pirki püchapeñi $\downarrow$ demorando", dijo el león.

32. velen piam konmatuy pañü $\rightarrow \mid$ 32. Así se les entró el sol. Entonces el ngürü veymu pepi piam nütulavi ti kusé zorro no pudo agarrar a la gallina vieja; achawall alkapio ume $\downarrow$ tampoco al gallo.

33. veymu püchapeñi ká trekakünowi $\downarrow \mid$ “-inchengechi umeen" pirki $\downarrow$

33. Entonces el león se puso otra vez en camino. "-Yo mismo voy a ir", dijo. veymu kiñe lawen ${ }^{31}$ yerki ta püchapeñi $\downarrow$ Entonces el león llevaba un remedio. veymu ngütrümpüllüpovi piam tati Llamó al alma de esa oveja. Pasó a ovisa $\downarrow$ | nürpoy piam kiñe llasurpoy agarrar, a lacear una oveja para hacerla püchapeñi kiñe ovisa ta kangkatupual $\downarrow$ asado. Hizo asado. Dicen que solito la kangkatuy piam $\downarrow \mid$ kidungechi trayivi amarró toda, la degolló y le comió la piam kom llovkentuvi imollviñvi $\downarrow \mid$ ngürü sangre $^{32}$. ¡El zorro no alcanzó allá! dipotulay $\uparrow \mid$

Entonces volvió a mirar atrás a su veymu ká wiñokintuki piam ñi malle sobrino el león. “-No alcanzó a (llegar) püchapeñi $\rightarrow \mid$ “- dip-palay nga weda aquí el muy bribón”, dijo. ngam-no” pirki $\downarrow$

34. vey ñochikechi piam inalli ngürü $\uparrow \mid$ “-lladkay may ñi malle nay" pilen 34. ¡Despacito iba siguiendo el zorro! amurki $\downarrow$ “-chumngechi chi ta

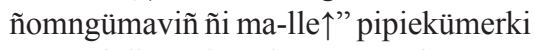
ngürü $\downarrow \mid$ dip-polay piam ngürü $\downarrow \mid$

"-Se va a enojar mi tío, pues", iba diciendo. “-¿Cómo amansaré a mi tío?", iba diciendo, mientras iba (caminando). Dicen que no alcanzó el zorro allá.

30 " [...] gamno [ngamno] huenthu $[$ wentru $]$ - hombre de poca importancia, de poca consideración, ò vil" (Febrés, Calepino: 492), "yamno, s. y adj., weda --: denuesto que significa "malvado inútil". Según otra interpretación* [en Panguipulli] no es ofensivo, se dice por travesura" (Augusta, Diccionario I: 54). El narrador lo tradujo por 'bribón'.

31 "l-awen, s., yerba medicinal; remedio cualquiera" (Augusta, Diccionario I: 111). ¿Poseía el león algún poder especial...?

32 Probablemente se sirvió ñachi, es decir, la sangre de la oveja coagulada y condimentada. 
35. vey umañkünupoy piam püchapeñi $\downarrow \mid 35$. Entonces se dispuso a alojar el menkuyey al-lümapu piam $\downarrow$ ká león. Llevaba carga lejos. Otra vez se umañkünu-poy kangkatopoy vey eppwe dispuso a alojar, hizo asado (y) después entuchillawi $\downarrow$ desensilló.

“-tüva mu umañan” pirki püchapeñi $\downarrow$ “-Aquí voy a alojar”, dijo el león. vey “-diküpakay tati” pirki püchapeñi $\downarrow \mid$ “-Tendrá que alcanzar aquí", dijo el león. püchi kimniekarki tayñi malle ñi Algo sabía cómo era su sobrino. chumngen $\downarrow$

36. emalkülepoki piam ngürü $\rightarrow \mid$ "-umagtule tayñi malle iñekontoviñ $\downarrow "$ pirki ngürü $\downarrow \mid$ kavey chem weda ngüñeli piam $\downarrow \mid$ amuy $\downarrow \mid$

36. Estaba cateando el zorro. "-Cuando se duerma mi tío me le voy a acercar", dijo el zorro. Dicen que también tenía muchísima hambre. Fue.

37. Itrokom kümelkünoy piam ñi 37. Dejó arreglado todito su cocaví rokiñ ta püchapeñi $\downarrow \mid$ veimu $\uparrow$ kudüy ta el león. Se acostó el león (y) durmió. püchapeñi umagtuy $\downarrow \mid$ vey umagtulu Mientras dormía, roncaba el león. külorinlu püchá-peñi $\downarrow \mid$ vey konpoy Entonces entró el zorro allá. “-Le voy piam ngürü $\downarrow \mid$ “-püchi weñeen ñi malle a robar un poco a mi tío", dijo el zorro. anü" pirki ngürü $\downarrow \mid$ mekepoy piam ñi Estuvo ocupado comiendo el zorro. in ngürü $\downarrow \mid$ lat-tikünowi piam ngürü $\downarrow \mid$ Quedó lleno el zorro. Le comió todas las vey kom iñmapovi piam ñi külche tripas a la oveja. Luego untó con caca de ti ovisa $\downarrow \mid$ vey piam $\rightarrow \mid$ re meovisa oveja la carne de su tío. Dicen que una künematuvi piam tayñi ilo tayñi malle $\downarrow \mid$ vez lleno, se retiró. Tenía miedo de que $\rightarrow$ wedalu ká iñetripatuy piam $\downarrow \mid$ llikay ñi se enojara.

lladkütungeal $\downarrow$

38. veymu $\uparrow$ ká epewün ká trepey 38. A la mañana siguiente despertó el püchapeñi ka kümeluwtuy $\downarrow \mid$ veymu león y se arregló. Entonces dijo: “-Me eypirki $\downarrow \mid$ “-weñe-panu nga tachi ngamno" vino a robar este bribón”, dijo el león. pirki ta püchapeñi $\downarrow \mid$ “-chumkünoaviñ $\uparrow \mid$ “-¿Qué le haré? Lo voy a azotar”, decía “trupoviñ” pirkevüy pü-chapeñi $\downarrow$ veymu el león. Entonces pensó otra vez el león. ká rakiduamüy püchapeñi $\downarrow \mid$ “-vemvili “-Si le hago eso, me dejará solo aquí”, nga kidukünotuanu nga tüva” pirki $\rightarrow \mid$ dijo. "-Mejor no lo hago así; es mejor “-doy kümi vemlaan piwkengen doy tener buen corazón”, dijo. kümi” pirki $\downarrow$

39. vey ká wiñoturki ñi malle $\downarrow \mid$ kümekange- pay piam ngürü $\downarrow$

39. Luego volvió su sobrino. Le buscó a la buena al zorro.

“-amuyu anü malle $\downarrow \mid$ "chem mu am ñam- "-Vamos amigo zorro, ¿por qué te tuymi $\uparrow ”$ pingerki ngürü $\downarrow \mid$ perdiste?", le dijo al zorro

-“-no nüvapetuviñ alka nga ti| püchi “-Bueno, estuve agarrando al gallo, pues. ngü-chatenu” pirki $\downarrow \mid$ "-itro kangkatoviñ Me insultó un poco". "-Pensé hacerlo piwün nga ti malle $\downarrow \mid$ perdonan anü asado, pues, tío”. “¡Perdóname amigo malle $\uparrow "$ pipingeturki ngürü $\downarrow \mid$ püchi tío!”, repetía el zorro. Dicen que el zorro trilalküli piam ngürü $\downarrow \mid$ estaba tirado un poquito de espaldas. 
40. ayey piam püchapeñi $\downarrow$ “-ya amuyu” pirki $\downarrow$ ká amurkingu $\downarrow \mid$ “-powtuli ruka mu ula vewla rüv elupotoviñ arreglo" pirki ta püchapeñi $\downarrow$
40. Dicen que se rió el león. “-Está bien, vámonos", dijo. Otra vez se fueron los dos.

“-Después, cuando llegue a la casa entonces sí lo voy a arreglar”, dijo el león.

41. vey $\uparrow \mid$ veychi pañü piam powi puel 41. Ese día llegaron a la Tierra del mapu $\downarrow$ yemi piam püt-ta kechami Oriente. Fue a buscar, a arrear hartos piam kullin ta püchapeñi $\downarrow$ küpalingu animales el león. (Los) trajeron. Dicen piam $\downarrow \mid$ vey meli antü ká küpatuingu que en cuatro días volvieron. Quedaba un piam $\downarrow \mid$ kiñe pañülewi ñi akutom tayñi día para que llegara a su tierra, entonces mapu mu püchapeñi $\rightarrow$ veymu “-ká dijo el león:

kiñe kangkatoayu anü malle $\uparrow$ eymi “-Hagamos otro asado amigo zorro, tú llovkentoymi anü” pingerki ngürü $\downarrow$ vas a degollar, amigo", le dijo al zorro.

42. vey ngürü chovürki|| ngürü $\downarrow \mid$ 42. Entonces le dio flojera... al zorro.

“-müt-te kutrankülen anü malle" pirki "-Estoy muy enfermo, amigo tío", dijo ngürü $\downarrow \mid$ podpodchili piam $\uparrow \mid$ vey chem el zorro. Dicen que estaba tiradito en el pilay piam püchapeñi $\downarrow$ vey: "kalli ||" suelo. No dijo nada el león. “- Déjenlo pirki "-to powtuli püchi ngülampotoviñ nomás...", dijo, "-cuando llegue allá ñi malle" pipingerki ñi piwke mu ta le voy a dar un poco de consejo a mi pücha peñi $\downarrow$. sobrino", repetía en su corazón el león.

43. vey kidu ká utrarki püchapeñi 43. Luego, él se levantó otra vez, volvió ka nüy kiñe (ovisa) ka llovkentuy ka a agarrar una oveja, (la) degolló e hizo kangkatuy $\downarrow$ asado.

“-chovülmi nay malle inche elulaayu ial "-Si tienes flojera, pues, sobrino, yo no nay $\downarrow$ ” pingerki ula (ngürü) $\downarrow$ “-chovün te daré comida”, le dijo después al zorro. ta elu ngekelay ial” pirki püchapeñi $\downarrow$ " “-A un flojo no se le da comida”, dijo el niküvüli piam ngürü $\downarrow$ león. Se quedó callado el zorro. “-ña kutralen malle $\uparrow ”$ piki piam ngürü $\downarrow$ “ “¡Estoy muy enfermo, tío!”, decía. trilal-leki piam $\downarrow$

Dicen que estaba tirado de espaldas.

44. vey upan ilu $\rightarrow$ vey ká umawtuy piam 44 . Después de comer de nuevo se $\downarrow \mid$ "-ule puliwen powtoyu” eypingerki durmió. “-Mañana temprano llegaremos ngürü $\downarrow \mid$ ká umawtuy piam püchapeñi $\downarrow$ allá (a la casa)”, le dijo al zorro. Se vey ká welukontuvi piam| ká weñevi durmió de nuevo el león. Entonces, dicen piam tayñi weñeñ patron piam nga ti que otra vez le entró (en su lugar), otra ngürü $\downarrow$ vez le robó a su patrón ladrón el zorro ${ }^{33}$.

33 El león no había sido un modelo de honradez en la Tierra del Oriente. 
45. veymu ula piawlngerki $\downarrow$

“-chumkaturkimi am anü malle $\uparrow$ eypingerki ngürü $\downarrow$ " "-to püchi wimapelayu powtuli nay $\uparrow " \mid$ pingerki ngürü ula $\downarrow \mid$ “-no anay malle $\uparrow ” \mid$ ká pikarki ngürü $\downarrow \mid$ “-ella kutrankülen anay malle” pipingeki piam $\downarrow$

46. vey $\uparrow \mid$ wün püle akutuy piam ruka mu $\downarrow \mid$ veymu akutulu ñi ruka mu vey ngütramka- patuy püchapeñi tayñi kure mu| eypinge- patuy tayñi llamngen $\downarrow \mid$ " -vey iawltenu nga iñ malle ayentuiawltenu reke nga vempi-iawltenu nga iñ malle| wedañma ngamno"| pirki püchapeñi $\downarrow \mid$

47. veymu lladkürki llamngen $\downarrow$ "-chumal ta nien ta weda trovün $\uparrow " \mid$ pirki llamngen $\downarrow$ | “-inche wemüentoviñ pirki $\downarrow \mid$ chovün inche ta duamngelan" $\downarrow$ vey veleki trukoli piam ngürü $\downarrow \mid$ “-chumkü--noanchi $\uparrow ”|~ p i k e r k i \downarrow|$

48. veymu travialu $\rightarrow$ utratripay piam püchapeñi $\downarrow$ tranalekerki ta ngürü $\downarrow$ veymu eypi piam $\downarrow$ “ “-püt-ta malle $\uparrow$ pi piam $\downarrow$ "-la-tolu inche ata anü malle $\uparrow "$ pirki ngürü $\downarrow$

“-chem pipimi anay $\uparrow ”$ pirkeyu ta püchapeñi $\downarrow$

“-latolu inche anü malle $\downarrow$ kimlan tüvachi travia upaanchi upalaanchi $\downarrow$ pepanu ñi kuku em cha ula $\downarrow$ " pirki ngürü $\downarrow \mid$ trilalleki ku- tranküli $\downarrow$

49. veymu vemngechilu piam wimanakümeyu ñi püllaw ${ }^{34}$ ta llamngen $\downarrow$ vey ñi püllawürke $\downarrow \mid$ vey $\uparrow$ tringkolkiawi piam ngürü pürakintuy piam wenumapu
45. Por eso, luego fue reprendido.

“-¿Qué te pasó, pues, amigo sobrino?” le dijo al zorro. " $-¡ T e$ voy a azotar un poco cuando llegue!", le dijo luego al zorro. “-¡No, pues, tío!”, decía el zorro. “-Estoy algo enfermo, tío", dicen que repetía.

46. Al alba volvió a su casa. Entonces, cuando volvió a su casa, vino a conversar el león con su mujer, la leona. "-Mi sobrino anda como riéndose de mí, me anda palabreando mi sobrino, malvado, bribón", dijo el león.

47. Entonces se enojó la leona.

“-¿Para qué tengo al flojonazo?”, dijo la leona. "-Yo lo voy a corretear", dijo; "-yo no necesito a un flojo".

Dicen que estaba así, echado, el zorro. “-¿Qué voy a hacer?”, decía.

48. Cuando anocheció, salió el león. El zorro estaba tendido. Entonces dijo: "¡Tío!", dijo, "-¡me voy a morir, estoy mal, amigo tío!", dijo el zorro.

“-¿Qué estás diciendo, pues?”, le dijo el león.

"-Me voy a morir, amigo tío No sé si voy a pasar o no esta noche. Me vino a ver recién mi finada abuela (paterna)", dijo el zorro. Estaba tendido de espaldas, estaba enfermo.

49. Así las cosas, dicen que le dio muchos varillazos su tía, la leona, ¡Era su tía! El zorro andaba revolcándose y miraba hacia el cielo. " $-¡$ Ven a buscarme,

34 Püllaw: esposa del tío paterno, en Cauñicú. La palabra no está consignada en los léxicos del mapudungu(n). 
ngürü $\downarrow$ “"-ye- yepatuan kuku个” pipingeki abuelita!”, decía una y otra vez el zorro. piam ngürü $\downarrow \mid$ vey wimangi piam $\downarrow \mid$ Le dieron varillazos.

-“müt'ay amuton kuku” ka piki piam $\downarrow \mid$ “-Lueguito me iré abuelita”, decía. Dicen pürakintuleki wenu piam $\downarrow$ que miraba hacia el cielo.

50. veymu püchapeñi eypirki $\downarrow \mid$ “-rüv 50. Entonces dijo el león: “-¿No será ku-tranlekapelay ñi malle $\uparrow$ ta pirki cierto que mi sobrino está enfermo? püchapeñi $\downarrow$ |“-latule ume vemkay tayñi ¡Póngale que se muera mi sobrino!”, ma-lle $\downarrow$ ” ta pirki püchapeñi $\downarrow$ dijo el león.

51. vey vemngechilu vey $\rightarrow \mid$ lay ula piam 51 . Así las cosas, luego se murió el zorro. ngürü $\downarrow$ pottiupay kümümüupoy piam Dicen que cayó al suelo (y) dio botes el ngürü $\downarrow \mid$ vey lay $\downarrow \mid$ vey kintuneweyu piam zorro. Se murió. Se quedó mirando a su ñi malle ta püchapeñi $\downarrow$ vey münayma mu sobrino el león. Durante un rato lo fue utungi piam ngürü $\downarrow \mid$ latuy piam ngürü $\downarrow \mid$ a mirar al zorro. Dicen que se murió el zorro.

52. veymu eypirki püchapeñi $\downarrow$ " “-latuy 52. Entonces dijo el león: “-Se murió mi nga ñi malle $\downarrow$ ”| veymu “-eymi nga sobrino, pues”. Luego: “-Por tu culpa, mi duam vente nga villpinman nga por tanto palabreo ${ }^{35}$, ahora se murió mi ñi malle vanten mu latuy" pingerki sobrino", le dijo a la leona.

püchallamngen $\downarrow$

"-chew ta lay weda koyla $\uparrow " \mid$ pirki ta llam-ngen $\downarrow$

"-iQué se iba a morir el muy mentiroso!", “-lay $\uparrow ”$ pirki $\downarrow$ ka kintulmeki piam ñi sobrino. Dicen que lo daba vuelta de una malle püchapeñi $\downarrow \mid$ tringkaymaiawlki pata, le agarraba una pata, daba vueltas piam namun mu piam nüki kiñe namun alrededor de él.

mu tringkolke-vüy piam $\downarrow \mid$

53. veymu $\rightarrow \mid$ ká utungeki piam $\uparrow \mid$ veymu ula piam perküntukukünotuy piam tayñi malle $\downarrow \mid$ nümüupay piam ñi perkün ngürü $\downarrow$ “-tüvi no vunalewetuy anchi个” este?", dijo el león. Se asustó mucho el pirki püchapeñi $\downarrow$ chem weda asustadowi león. ¿Qué voy a hacer, pues?”, dijo. piam ta püchapeñi nga $\downarrow \mid$ “chumküno nga “-¿Cómo resucitaré a mi sobrino?”, dijo ta $\uparrow "$ pirki|“"-chum-ngechi nga mongelton llorando.

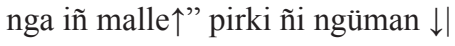

54. veymu ngümawturki püchapeñi $\downarrow$ 54. Entonces lloró el león. “-Oyoyoy, “-oyo-yoy oyoyoy oy oy" pirki ñi oyoyoy, oy, oy”, lloraba el león, sintiendo ngüman püchapeñi| ngümanpelu pena por su sobrino muerto. weñangkülu lalu tayñi malle $\downarrow \mid$ 
55. veymu $\uparrow \mid$ "-wichamachian" pirki $\downarrow \mid$ 55. Entonces dijo: "-Voy a invitar a un “-kin-ton machi machitual tayñi malle $\downarrow \mid$ machi, para que le haga machitun a mi mongel-ton" pirki püchapeñi $\downarrow \mid$ veymu ka sobrino. Lo haré resucitar”, dijo el león. ngütrümüy piam kiñe peñi ka niekerki $\downarrow \mid$ Entonces llamó también a un hermano veymu ramtungerki “-chumkülimi nay que tenía. Le preguntó: “-¿Cómo estás, püt-ta unen $\uparrow$ pingerki $\downarrow \mid$ vey “-kimlaymi hermano mayor?”, le dijo. Luego:

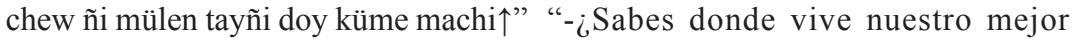
pingerki pücha unen $\downarrow \mid$ machi?", le dijo al hermano mayor.

56. veymu eypirki püt-ta unen $\downarrow \mid$ "inche 56. Entonces dijo el hermano mayor: nien "-Yo tengo un sobrino materno", dijo, kiñe choküm anü” pirki $\downarrow$ |"-mollko mapu “-vive en la tierra de Mollco”, dijo, müli” pirki $\downarrow$ “"-veymu metu ehtudiay $\downarrow \mid$ “ahí está estudiando. ¡Es un excelente vey tuttechi machi $\uparrow "$ pirki $\downarrow \mid$ machi!", dijo.

“-ñi pingi anchi个 pirki püchapeñi $\downarrow$ “-¿Cómo se llama?”, dijo el león. vey ta| “-walkipayun ${ }^{36}$ pingi tayñi üy” “-Se llama Hualquipayún”, dijo. “¡Es pirki $\downarrow$-“vey ñaykirke” $\downarrow$

-“kintoviñ" pirki $\downarrow$ un gato!".

“-Lo voy a buscar", dijo.

57. vey piam trawluingün nga ka 57. Entonces se juntaron y llamó a cada ngütrümüy piam kake pu ngürü piam uno de los zorros, sus parientes. Dicen tayñi pu piwke $\downarrow^{37} \mid$ püt-ta trawiñmangi que se le juntaron muchos. Entonces piam $\downarrow \mid$ veymu piam akuy rakiñ $\downarrow^{38} \mid$ akuy llegó ahí la bandurria. Llegó riéndose a piam rakiñ veymu $\downarrow$ püt-ta ayepay piam carcajadas la bandurria. El zorro estaba rakiñ $\downarrow \mid$ ngürü trilalpürali veymu kay $\downarrow \mid$ tendido en el suelo ahí.

“-ja ja ja ja ja ja $\uparrow$ pirki rakiñ $\downarrow$

“-¡Ja, ja, ja, ja, ja, ja, ja!”, dijo la veymu piam epe nengümuwüy piam ta bandurria.

ngürü $\downarrow \mid$ epe ayetuy piam ngürü $\downarrow \mid \quad$ Entonces casi se movió el zorro. Dicen que casi se rió el zorro.

58. vemngechilu vey ta $\rightarrow \mid$ "-amon” 58. Así las cosas, “-Voy”, dijo el león. pirki püchapeñi $\downarrow \mid$ “-kinton $\downarrow ” \mid$ nükolluy “-Voy a buscar(lo)”. Agarró caballo el piam püchapeñi kintolu ñi choküm $\downarrow \mid$ león, para buscar a su sobrino. Se puso amukünowi piam $\downarrow$ mollkomapu en camino. No se sabe donde queda la kimngelay chew ñi ngevel $\downarrow$ tierra de Mollco.

36 Quizá el nombre era originalmente Waykipayun 'bigotes de lanza'.

37 Piwke significa 'corazón', pero en el relato, 'pariente'. Otros animales, incluidos los pájaros, son parientes del zorro.

38 La bandurria (Theristicus melanopis), ave común en los campos, suele reirse a carcajadas en los epew que hemos oído. Curiosamente, se ríe como en español, con el fonema /x/que el mapudungu(n) no posee. 
59. velen amuluy püchapeñi $\uparrow \mid v e y m u \uparrow$ chal- amulu $\rightarrow \mid$ veymu tragürki kiñe sañi $\downarrow^{39} \mid$

"-chew amoymi nay pücha unen $\uparrow "$ pinge- rki $\downarrow$ “-kinton kiñe machi $\downarrow$

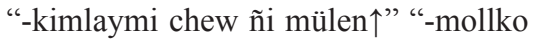
mapu pi piam müli $\downarrow \mid$ “-walkipayun pingi piam ñi üy” pirki $\downarrow$ | "tiechipüle püt-ta mawida mu müli| veymu müli” pirki sañi $\downarrow$ ká amukünowi piam $\downarrow \|$ ká trekakünowi encaminó entonces hacia allá. piam veymu tiepüle $\rightarrow \mid$

60. ká tragi piam kiñe killkill $\downarrow^{40}$ 60. Dicen que también encontró a un “-marimari nay killkill” pirki püchapeñi $\downarrow$ quil-quil. “-¡Buenos días, quilquil!”, “-marimari pücha unen” pingerki dijo el león.

püchapeñi $\downarrow \mid$

“-kimlaymi chew ñi mülen tayñ Walkipayun $\uparrow ”$ pingerki killkill $\downarrow$ “-mollko mapu müli” pirki $\downarrow \mid$ "-metu allü-tripali” pirki $\downarrow$

59. Así, se fue el león. Cuando iba un poco lejos, encontró a un chingue.

“-¿Adonde vas, pues, hermano mayor?", le dijo. "-Voy a buscar a un machi. ¿Sabes donde vive?" “-Vive en la tierra de Mollco. Dicen que se llama Hualquipayún", dijo. "Por allí, en la montaña grande, vive. Ahí vive”, dijo el chingue.

Dicen que otra vez se fue el león. Se

“-Buenos días, hermano mayor", le dijo al león.

“- ¿Sabes donde vive nuestro Hualquipayún?", le dijo al quilquil.

“-Vive en la tierra de Mollco", dijo. “-Todavía está lejos”, dijo.

61. vey ká trekakünowürki $\downarrow \mid$ ká amuy 61. Otra vez se puso en camino, otra piam püchapeñi $\downarrow \mid$ püchiken ngümaki vez se fue el león. Dicen que lloraba un piam pücha-peñi $\downarrow$ poco el león.

vey ngürü vaw tayñi ruka mu püt-ta Aquí en su casa se habían reunido trawi-ñmanengi piam $\downarrow$ muchos alrededor del zorro.

62. vemngen ká konpoy piam kiñe püt-ta 62. Así las cosas, otra vez entró en lemuntu mu $\rightarrow \mid$ veymu ká tragürki kiñe un monte grande. Entonces encontró kowkow $\downarrow^{41}$ también a un coucou.

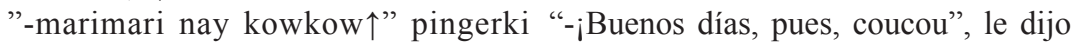
kow-kow $\downarrow$ al coucou. "¡¡Buenos días, hermano

39 Otro nombre del chingue (Conepatus chinga), un mustélido cuyos orines son extremadamente fétidos.

40 Este búho (Glaucidium nanum) es denominado también chonchón y tuétué. Se lo relaciona con la brujería.

${ }^{41}$ Es el búho tucúquere (Bubo virginianus), llamado tambien coucou y toutou. 
“-mari mari püt-ta unen" pirki kowkow $\downarrow$ mayor!”, dijo el coucou. “-¡Ji, ji. Ji, “-ji ji ji ji ji ji ^”püt-tü piam ayey ji, ji, ji!”, dicen que se rió, con el pico kowkow $\downarrow$ fruncido, el coucou.

“-ayeymi nay püt-take nge” pingerki “-¡Te ríes, pues, ojos grandes!”, le dijo kow-kow $\downarrow$

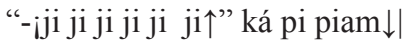
vemkünokakerki ka amuy piam $\downarrow \mid$ al coucou. “-¡Ji, ji, ji, ji, ji, ji, ji!”, volvió a decir. Dicen que lo dejó así y se fue.

63. veymu个 powi piam ti püt-ta mawida 63. Entonces llegó a la montaña grande el mu ta püchapeñi $\downarrow \mid$ veymu powlu ti püt-ta león. Cuando llegó a la montaña grande, mawida $\mathrm{mu} \rightarrow \mid$ veymu piam ká tripapay entonces dicen que salió también la piam ta || $\mid$ vihkacha $\downarrow^{42} \mid$ veymu nierki ñi vizcacha. Ahí tenía su casa.

ruka $\downarrow \mid$ "“-marimari nay wenüy $\uparrow ” \mid$ pingerki “-¡Buenos días, pues, amiga”, le dijo a vih-kacha $\downarrow$ la vizcacha.

“-marimari püt-ta unen $\downarrow$ ”| pingerki “-Buenos días hermano mayor”, le dijo püchape- ñi $\downarrow$ al león.

64. veymu $\uparrow$ “-kimalami ruka ta Walkipa- 64. Entonces “-¿Conoces la casa de yun $\uparrow$ " pirki püchapeñi $\downarrow$ Walkipayún?", dijo el león.

“-tüva mu müli anü tüvi mu vurüpüle| “-Aquí vive, amigo, ahí detrás tiene la veymu nii ruka $\downarrow$ ”| veymu piam kintuli casa”. Entonces miró su reloj la vizcacha. ñi ora piam vihkacha $\downarrow \mid$ “-tüva mu “-Ahora está durmiendo, amigo”, dijo, umagli anü” pirki $\downarrow \mid$ “-ñochikechi “-entra despacito donde él”. kontupowimi $\downarrow " \mid$

65. vey amuy piam $\rightarrow$ ñochikechi 65. Fue el león. Entró despacito el konpoy püchapeñi chew iñ mülemom león donde estaba el machi... el gato. tati machi ||tati ñayki $\downarrow$ umagli piam Dicen que estaba durmiento el gato (y) ñayki $\uparrow$ kongkülmengi piam $\downarrow \mid$ veymu roncando. Entonces se acercó a mirar adkintungepoy piam ñayki $\downarrow$ al gato.

“-tre|pelimi nay toküm $\uparrow$ pirki pücha- “-¿Estás des...pierto, pues, sobrinito?”, peñi $\downarrow$ dijo el león. “- ¡J!”, dijo el gato. Se “- $\mathrm{j} \uparrow$ !” pirki ñayki $\downarrow \mid$ lladküy piam ñayki $\downarrow \mid$ enojó el gato.

vey "-lladkükelmi anay toküm| na weda "-No te enojes, pues, sobrinito. Traigo un dungu küpalün nay”| pirki püchapeñi $\downarrow \mid$ asunto muy grave”, dijo el león.

66. veymu ñochikangepoy piam tati 66. Entonces le buscó a la buena al machi $\downarrow$ machi.

“-duam mu küpan anü toküm” pingerki “-Vine por una necesidad, pues, amigo ti machi $\downarrow \mid$ "-lalkatun may ñi malle em $\uparrow \mid$ sobrinito", le dijo al machi, “-ipor la

${ }^{42}$ La vizcacha (Lagidium viscacia) tiene también nombre mapuche: truwi. El nombre vizcacha es de origen quechua. 
dew-ma vunaletuy $\downarrow \mid$ veymu yepapeyu muerte de mi finado sobrino (paterno)! nga tüva $\downarrow$ püchi lawentulmeatuanu Ya se está pudriendo. Por eso te vine a pipen anü toküm" pingerki ti ñayki tati buscar ahora. Pensé, pues, que me irías machi $\downarrow$

a hacer un poco de remedio, amigo sobrinito", le dijo al gato, al machi.

67. niküvuli piam ñayki $\downarrow \|$

“-veyllepu $\uparrow$ pirki al-lüñma mu $\downarrow$

"-chem ta kopuralmi kulliayu| chem ta duamngelmi $\mid \rightarrow$ kom trawlelayu $\downarrow \mid$ pirki

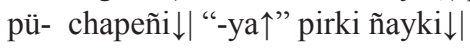
“-chem wema ipual kom eypian” pirki $\downarrow$ “-ya $\uparrow$ pirki ñayki $\downarrow$

68. veymu "- trawlelmon konna" pirki ta ñayki $\downarrow$ " “-wemaldungu kintulmon ta trupolu kultrung” pirki $\downarrow \mid$ veymu “-ká kintulmon tati tayelalu” ká pirki $\downarrow$ veymu "-ká kintulmon nga ti kavavaalu" pirki $\downarrow$ “-tuputrupotolu nga kultrung ká kintoymün” pingerki $\downarrow$ | "-vey nga inche nga ñi ial vey nga ñi ül mo eypiayu” pingerki püchapeñi $\downarrow$ “-ya $\uparrow$ müt’ay amon” pirki $\downarrow$

69. “- wemaltu wema ta pewuton" pirki ta ñayki $\downarrow \mid$ “-veymu kimaviñ mongeal mo- ngenoal $\downarrow$ ”| "-mongey pieli mu ñi metu iñ ki- melkaadu rangiñpañüpual" pirki ta nayki $\downarrow$ “-ya $\uparrow ”$ pirki püchapeñi $\downarrow \mid$ wiñotuy piam trawülchepatolu $\downarrow \mid$
67. Dicen que se quedó callado el gato. “-¡Está bien!”, dijo después de un rato. "-Lo que cobres te lo pagaré, lo que necesites, todo te lo reuniré", dijo el león. “-¡Está bien!”, dijo el gato.

“-Lo que comerás primero, todo me lo dirás”, dijo. “¡¡Está bien!”, dijo el gato.

68. Entonces: “-Reúnanme mocetones", dijo el gato. "-En primer, lugar búsquenme al que va a tocar el tambor". "-Búsquenme también al que va a cantar tayel"43, dijo. En seguida: "-Búsquenme también al que va a gritar kavavan" 4 , dijo. "-Busquen también al que va a tocar el tambor"45, le dijo. "-Mi comida te la diré en mi canto”, le dijo al león. “-¡Está bien!, voy en seguida", dijo

69. "-Primeramente voy a adivinar", dijo el gato. "-Entonces sabré si vivirá o no". "-Si el que me está enseñando me dice que va a vivir, llegaré allá al mediodía", dijo el gato.

“-¡Está bien!", dijo el león. Dicen que volvió, a reunir gente.

43 Probablemente la palabra, en la forma tayül, ha sido empleada originalmente en ceremonias de machitun y ngillatun, en Argentina, por ejemplo, en Neuquén. Está consignada en las obras de Wily Hassler y Rodolfo Casamiquela (en la de este en la forma taiël), y traducida como 'canto religioso', 'canto sagrado'

44 Kavavan: en Cauñicú, gritos proferidos con el fin de alejar a los malos espíritus que merodean cerca del espacio ritual del ngillatun, y también para animar, para despertar el inamongen (espíritu, alma), "kefafan, n., interrumpir la voz dando palmoteos, contra la boca abierta, con lo cual los indígenas expresan su regocijo en sus rogativas, al vencer en sus juegos, etc." (Augusta, Diccionario I: 80).

45 Insiste en la presencia de quien toca el kultrung, seguramente por la importancia del instrumento en la ceremonia. 
70. akutuy piam $\downarrow \mid$ akutulu veymu $\rightarrow$ püt-ta trawli piam konlepowi nga ti pu konna $\downarrow \mid$ veymu nga eypipaturki $\downarrow \mid$ “-eypienu nga iñ choküm” pirki $\rightarrow \mid$ "-wemaldungu mu nga kintulgen nga trupolu kultrung pingen tata $\downarrow$ " pipaturki ñi kure püchapeñi $\downarrow \mid$ “-ñi ta yavuluavüychi $\uparrow$ pirki. $\downarrow \mid$ veymu eypirki killkill "inche yavuluan tru- polu kultrung $\downarrow "$

71. veymu ká eypingen veyta $\rightarrow$ “- ká 71. “-Se me dijo además: “-Búsquenme kin- kintulngen nga tüvi ti tayelalu $\rightarrow$ también al que va a cantar, al cantor

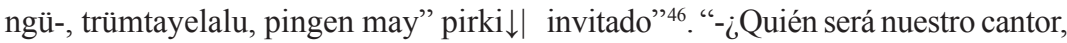
“-¿¿ñi nga iñ tayel ñi nga iñ yavuluay quien le hará empeño ahí?", dijo el león. chi veymu个" pirki püchapeñi $\downarrow \mid$ "-inche "-Yo le voy a hacer empeño", dijo el yavuluan" pirki lavatra $\downarrow \mid$ vey $\rightarrow \mid$ sapo. vemngechi piam $\downarrow \mid$

72. veymu nga ká kintulngen nga tüva en-tukavavaalu” pirki $\downarrow$ " “-ñi chi nga ká yavuluavüy $\uparrow "$ ká pirki püchapeñi $\downarrow \mid$ veymu triwkü ${ }^{47}$ miawürki piam $\downarrow \mid$ -“-inche yavuluan anü kompañ $\uparrow "$ pirki chi triwkü $\downarrow$

vey pey piam kom ñi duamngen $\downarrow$

73. vey $\rightarrow||$ "“-ká duamngen nga kiñe traw- 73. "-Necesito también a uno que reuna leladu nga||nga kay iñ kangkantupual nga (cosas)... para que hagamos el asado a tayñ machi" $\downarrow$ "ñi chi nga yavuluavüy nuestro machi”, dijo el león. “-iQuién nga iñ trawleladu $\uparrow$ pirki ta püchapeñi $\downarrow \mid$ le haría empeño para reunir(las)?", dijo veymu eypirki ñamku $\downarrow^{48} \mid$ “-inche nga el león. Entonces dijo el aguilucho. trawlelayu chem chi yelmeayu" pingerki "-Yo te reuniré cualquier cosa, yo te püchapeñi $\downarrow$ “-kiñe mula yelmean anü” la iré a buscar", le dijo al león. "-Voy

46 Ngütrüm: el 'invitado' que actualmente suele proceder de otra comunidad, para participar en un baile o, como en el relato, para cantar.

47 El tiuque o triuque (Milvago chimango), ave falconida muy común en los campos (la cual actualmente se ve también en ciudades, por ejemplo, en Santiago). En algunos epew es considerado un individuo de baja categoría social (weya wentru).

48 El ñamku (Geranoaetus polyosoma) es un ave de categoría. Suele encarnar también el espíritu de un antepasado. 
pingerki $\downarrow$ tripay piam ñamku kintolu a buscar una mula, amigo", le dijo. mula $\downarrow \mid$ veymu piam yemi kiñe püt-ta Salió el aguilucho a buscar una mula. dewü piam ñamku $\downarrow$ akulüy piam kiñe Entonces fue a buscar un ratón grande püt-ta dewü ipayal ti machi $\downarrow \|$ el aguilucho. Dicen que trajo un ratón grande, para que comiera el machi.

74. veymu wünpüle $\uparrow$ akuy piam| 74. Entonces, en la mañana, al medio rangiantü piam akuy ñayki $\downarrow \mid$ püllki día dicen que llegó el gato. Pasó con la ñi külen piam upay tripapay lemu cola paradita, salió del monte. Caminaba mu $\downarrow \|$ ñochikechi trekalli piam upay $\downarrow \|$ despacito. Pasó. “-¡Asado, asado,

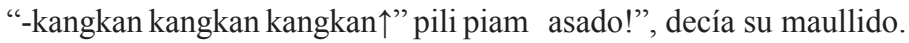

tayñi ñawawan $\downarrow$

“-küpay” pingerki $\downarrow \|$

"-Vino", dijeron.

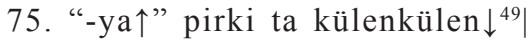
ngüchal- konnarki kavavalngeal nga ti||. Veymu triwkü| "-triw triw triw triw triw $\uparrow ”$ pirki triwkü ñi kavavan $\downarrow$ kavavan mu nga llowngi piam tati triu". machi $\uparrow \mid$ vey $\uparrow$ tragmengi piam $\downarrow \|$

75. “-¡Listo!”, dijo el "cola de palo”. Animaba a los mocetones para que gritaran kavavan. Entonces gritó su kavavan el tiuque: "-triu-triu-triu-triu-

¡Dicen que fue recibido con kavavan el $m a c h i$ ! Fueron a encontrarlo.

76. üngküupay piam ti kutran mu ti 76. Pasó derechito el machi donde el machi tati la mu $\downarrow$ vey akulu anülleki enfermo, donde el muerto. Cuando piam ñayki $\downarrow \mid$ pürakintuleki wenumapu $\downarrow \mid$ llegó, se sentó un rato el gato. Miraba el veymu eypi-rki $\rightarrow \mid$ "-mongey $\uparrow ”$ pirki cielo. Entonces dijo: “-iVa a vivir!”, dijo ñayki $\downarrow \mid$ veyta $\rightarrow \mid$ "-ñaykurewen ${ }^{50}$ may el gato. "-Falta que se instale el rewe, valtay" pirki $\downarrow$ “-yel-metoviñ nga püllü pues”, dijo. "-Voy a ir a buscar el alma wenumapu” pirki ñayki $\downarrow$ "“- nagpatulay al cielo", dijo. "-No ha bajado su alma. ñi püllü $\downarrow$ trawütoy ñi kural-nge” pirki Va a cerrar su pupila”, dijo el gato. nayki $\downarrow$

77. veymu kaveleki piam ti ñayki $\downarrow$ 77. Entonces estaba así el gato. “-¿Qué “-chem lawen chi nga eloviñ nga remedio le daré a este enfermo?", decía tachi kutran ” pikerki ñayki $\downarrow$ veymu el gato. Entonces pensó. El gato ya rakiduamürki $\downarrow \mid$ vey ti ñayki kimnekarki conocía la astucia del zorro. "“-Voy a ir a

49 El cola de palo (Falco sparverius) es un ave falconida conocida comúnmente con el nombre de cernícalo.

50 Ngeykurewen -como se suele denominar- es la ceremonia de iniciación del (de la) machi, en la cual instala o renueva su rewe (árbol sagrado). Quizá -como todavía estaba estudiando-era la primera actuación públiva de Walkipayun. La palabra significa literalmente 'mecer el rewe'. 
tayñi ngünenngen ti ngürü $\downarrow$ “-1lepiñ buscar hormigas”, dijo el gato, dijo solo; yemen” pirki ñayki| pirki kidu eypilay no dijo nada (en voz alta). ume $\downarrow$

78. vey ñaykurewey piam $\downarrow$

“-trupulngean kultrung pi piam $\downarrow$ veymu nüy piam kultrung ta killkill $\downarrow \mid$

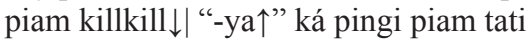
tayelve $\downarrow^{51} \mid$ eluy piam tayñi tayel lavatra $\downarrow \mid$ chem kümelkay piam ñi tayel lavatra $\downarrow$ veymu tayeli piam lavatra $\downarrow \mid$

79. meki piam ñi kavaban ta triwkü $\downarrow \mid$ vey $\rightarrow$ ñamku kay vey piam eluy ta ilolu ñi mula $\downarrow$ avkentu trupulngi kultrung piam ti kavavan meki piam machitun $\downarrow \|$ vey piam "-traw traw traw traw traw" pi

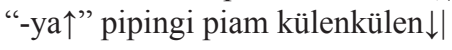

78. Entonces instaló el rewe.

"-Tóquenme el cultrún", dijo.

Entonces agarró el cultrún el quilquil.

"-¡Trau-trau-trau-trau-trau!", dijo el quilquil.

“-¡Listo!”, le dijeron también al cantor.

Comenzó su canto el sapo. Dicen que cantó muy bonito el sapo. Cantó el sapo ahí.

79. El tiuque estaba ocupado gritando kavavan. Entonces el aguilucho comenzó a carnear su mula. Tocaron harto el cultrún, gritaron kavavan, hicieron el machitún.

“-¡Listo!", dicen que repetía el "cola de palo".

80. veymu pürakintuy piam ñayki $\rightarrow$ trawtuy piam ñi kuralnge $\downarrow \mid$ veymu eypi piam tati pu konna "-mongetoy nga tachi kutran" piwürkingün ka trepelkawingün piam $\downarrow \mid$

80. Entonces, dicen que miró hacia arriba el gato, cerró su pupila. Entonces se decían unos a otros los mocetones: "-Va a resucitar este enfermo", se decían y se alegraban.

81. veymu vemngechilu $\rightarrow \mid$ ampüray 81 . Entonces, así las cosas, levantó su piam ñi lipang ti machi $\downarrow \mid$ vey niküvnagi brazo el machi. Se callaron todos, los piam ti pu kavave tayelve kom $\downarrow$ que gritaban kavavan, el cantor, todos. rakiduamnkülewi piam $\downarrow$

Dicen que se quedó pensando.

82. veymu piam eluy piam ülkatolu ta 82 . Entonces comenzó a cantar el gato, el ñay-ki tati machi $\downarrow$ "“-kangkan kangkan machi. "-¡Asado, asado, asado, asado!”,

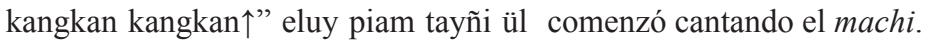
tati machi $\downarrow$ vey "-kangkatoyu|| kangkatolngey”, dijo el león. pirki pücha peñi $\downarrow$

51 En el relato tayelve significa 'cantor'. En el ngillatun que se celebra en Cauñicú se denomina así al coro de mujeres que participa en al ceremonia, es decir a una pluralidad de personas. 
83. veymu rakiduamleki $\rightarrow$ piam ñayki $\downarrow \mid$ 83. Entonces estaba pensando el gato. nümünümütuy nay ñi pilun ta ngürü $\downarrow \mid$ Le olfateó, pues, la oreja al zorro. Dicen aye-leki piam ti machi $\downarrow \mid$ veymu piam que se reía el machi. Luego sacó una entupay külen kollu| tukulelngi ñi yu cola de caballo (y) se la puso en la nariz mu ngürü $\downarrow$ nagikochalmangi piam ñi al zorro. Le hizo cosquillas en la nariz. $\mathrm{yu} \rightarrow \mid$ echiwtul- ngi $\downarrow \mid$ vey vemngechilu Lo hizo estornudar. Cuando ocurrió

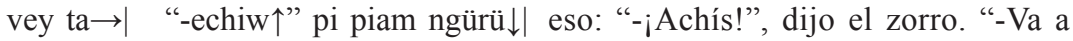
“-mongey” pingerki $\downarrow \mid$ vey ká tukulelngi vivir", dijeron. Otra vez le pusieron; no piam $\downarrow$ echiwwelay $\downarrow \mid$ “-mo-ngey” pirki estornudó. “-Va a vivir”, dijo el gato. ta ñayki $\downarrow$

84. veymu ká eypirki $\rightarrow$ “-ka kiñe lawen 84. Entonces volvió a decir: “-Hay otro müli yemeen” pirki ñayki $\downarrow$ " “-püchi remedio, voy a ir a buscar(lo)", dijo el üngel-mon” pirki $\downarrow \mid$ ká amuy piam gato. “-¡Espérenme un poquito!”, dijo. lemu mu $\downarrow$ veymu yemerki ta llepiñ $\downarrow \mid$ Fue otra vez al bosque. Entonces fue a chemuvün llepiñ kellü llepiñ piam buscar hormigas. Dicen que trajo varias küpalüy ti mamüll llepiñ nga $\downarrow$ “-llepiñtuñmaviñ liwe ${ }^{52}$ " pirki $\downarrow$ hormigas coloraditas, hormigas de la madera.

"-Le voy a poner hormigas en el ano", dijo.

85. vey chem münayma mu piam akutuy 85 . Dicen que al poco rato volvió el piam ti machi $\downarrow \mid$ ká echiwtulngepay machi. Otra vez lo hizo estornudar (al piam $\downarrow \mid$ veymu entupay kiñe llepiñ $\rightarrow \mid$ zorro). Entonces sacó una hormiga y se la üpürtuku-künolgi piam ñi liwe mu $\downarrow$ dejó puesta en su ano, para que le picara. nükov- künovemüy piam ñi poto ${ }^{53}$ De repente encogió el poto el zorro.

ngürü $\downarrow$

“-mongey” pirki ñayki $\downarrow$

"-iVa a vivir!", dijo el gato.

Otra vez lo hicieron estornudar. Le ká echiwtulngi piam $\downarrow \mid$ udañmangeki abrieron, los ojos al zorro. No se abrieron. piam ñi nge ngürü $\downarrow \mid$ udakelay nga $\downarrow \mid$

86. ká echiwtulngi vewla ká echiwi 86. Otra vez lo hiceron estornudar; ngürü $\downarrow \mid$ veymu eypirki ti machi $\rightarrow \mid$ ahora estornudó el zorro. Entonces dijo “-püchi doy tukulelaviñ lawen",pirki $\downarrow \mid$ el machi:

“-epe akutuy ñi püllü $\downarrow "$ "-tüva tayñi “-Le voy a poner otro poco de remedio", inamongen tati kon- tolu” pirki ti machi $\downarrow$ dijo. "-Casi volvió su espíritu”. “-Ahora pengeli piam kellüke llepiñ $\downarrow \mid$ veymu va a entrar de nuevo su alma”, dijo el

52 “liwe el ano, el sieso" (Alonqueo 1989: 141).

53 "Poto - el siesso" (Febrés, Calepino: 600), "Poto, sedes, anus" (Havestadt, Chilidúǵu II: 754). 
piam tukulelngi meli llepiñ piam ñi liwe machi. Dicen que mostró hormigas mu $\downarrow$ ngüñomngi piam $\downarrow \|$ coloraditas. Entonces le puso cuatro ${ }^{54}$ hormigas en su ano. Dicen que le pusieron una cala.

87. püchiñma piam velewekawi 87. Un ratito se quedó así, aguantó el awan- takawi piam ngürü $\downarrow \mid$ alüñma zorro. Al ratito, de repente se encogió mu piam nükovnükovkünovemüy varias veces. Le dolía su ano. Al poco piam $\downarrow \mid$ münañma mu rüngkülen rato salió saltando el zorro, pues. Dicen tripay piam nga ngürü $\downarrow \mid$ kutrantuli ñi que andaba dando grandes saltos (de un liwe $\downarrow \mid$ püttake rüngküyawi piam $\downarrow \mid$ lado para otro). "-Agárrenlo, agárrenlo, "-nüvimün nüvimün nüvimün pi piam agárrenlo", dijo el machi. Se juntaron a ti machi $\downarrow$ trawiñmangi piam veymu $\downarrow \mid$ su alrededor ahí.

“-mongetuy nga ti” pirki ti machi $\downarrow$ " “- Volvió a vivir, pues!", dijo el machi.

88. mongetuy piam ngürü $\downarrow$ püt-ta 88 . Dicen que volvió a vivir el zorro. trawiñmangi veymu $\downarrow$ itrokom nüngetuy Se juntó harta gente a su alrededor. Lo piam $\downarrow \mid$ veymu $\downarrow$ "-wiñoymi am nay agarraron enterito. Entonces: “-¿Así que malle $\uparrow$ " pirki ñi malle ta püchapeñi $\downarrow \mid$ “"- volviste, amigo sobrino?", le dijo a su wiñotun anü malle" pirki $\downarrow$ sobrino el león. "-Volví, amigo tío", dijo.

89. veymu piam püchi lladkütuy ta 89 . Por eso, dicen que se enojó un poco ngürü $\downarrow$ el zorro. "-Se burló de mí este hombre, "-ayentenu nga tachi wentru| tukulelenu pues. Me puso hormigas en mi ano", nga llepiñ ñi liwe mu" pirki ngürü $\downarrow \mid$ müt- dijo el zorro. Se enojó mucho, se enojó te lladküy piam lladkütuvi tati machi $\downarrow \mid$ con el machi.

90. veymu lladkülu trukuel ngürü $\rightarrow 90$. Cuando parecía enojado el zorro, veymu piam püt-ta ayeñeyu ta rakiñ $\downarrow \mid$ dicen que se rió a carcajadas de él la “-ja ja ja ja ja ja $\uparrow$ ” pi piam rakiñ $\downarrow$ bandurria.

doy lladküy piam ngürü $\downarrow$ “-ayenetenu “-iJa, ja, ja, ja, ja,ja, ja!”, dijo la bandurria. nay kelü matra" pingi piam rakiñ $\downarrow \mid$ Más se enojó el zorro. "-Me embromó, veymu piam doy lladkütuy ta ngürü $\downarrow$. pues, la patas coloradas", dijo de la bandurria. Por eso dicen que más se enojó el zorro.

91. vey müt'ayno mongetulukay tati $\downarrow$ 91. Pronto volvió a vivir, pues. Volvió a mo-ngetuy ngürü kom tayñi chumleken vivir el zorro y a ser como siempre era. ká vele- tuy $\downarrow \mid$ welu kiñe antü $\rightarrow \mid$ "-itro Pero un día dijo: "-Lo voy a joder", le waltalaviñ" pirki pirkevi ta ñayki $\downarrow$ dijo al gato. 
92. veychi piam ta yavkawtüy ta ñayki 92. Dicen que en ese tiempo se engu ta ngürü $\downarrow \mid$ veymu wanten mu ta| disgustaron el gato y el zorro. Por eso ngürü chew upey ñayki| veykachi ta ahora, donde el zorro encuentra al gato nüvawnüvaw-ngetuki $\downarrow \mid$ veymu piam en seguida siempre lo persigue. Por eso tati ngütram $\downarrow$ dicen la conversa.

\section{REFERENCIAS BIBLIOGRÁFICAS}

Alonqueo, Martín. 1989. El habla de mi tierra. Mapudungun. P. Las Casas, Temuco: Ediciones Kolping.

Augusta, Félix José De. 1916. Diccionario Araucano-Español y Español Araucano. Santiago: Imprenta Universitaria.

Febrés, Andrés. 1765. Arte de la Lengua General del Reyno de Chile, con un Diálogo ChilenoHispano muy curioso: a que se añade la Doctrina Christiana, esto es, Rezo, Catecismo, Coplas, Confesionario, y Pláticas, lo más en Lengua Chilena y Castellana: Y por fin un Vocabulario Hispano Chileno, y un Calepino Chileno Hispano más copioso. Compuesto por el P. Andrés Febrés Misionero de la Com. de Jesús. Año de 1764. [...] Con Licencia: en Lima, en la calle de la Encarnación.

Havestadt, Bernardo. [1777] 1883. Chilidúǵu sive Tractatus Linguae Chilensis opera Bernardi Havestadt. Editionem novam inmutatam curavit Dr. Julius Platzmann. Vol. I y II. Lipsiae: in aedibus B.G.Teubneri.

Lenz, Rodolfo. 1895-1897. Estudios Araucanos [...]. Santiago: Imprenta Cervantes.

SÁnChez, Gilberto, 1989. Relatos orales en pewenče chileno. Anales de la Universidad de Chile [5. Serie] 17: 289-360.

1996. Relatos orales mapuches (procedentes del Alto Bío-Bío, VIII Región). Boletín de la Academia Chilena de la Lengua 71: 289-301.

2003-2004. Vida de un pewenche. Lorensu Nawpa tayñi mongen. Revista Chilena de Antropología 17: 183-198.

2010. Los mapuchismos en el DRAE. Boletín de Filología XLV(2): 149-256.

VAldivia, Luis DE. 1606. Arte y Gramática General de la Lengva Que corre en todo el Reyno de Chile, con vn Vocabulario, y Confessonario. Compuestos por el Padre Luys de Valdivia de la Compañia de Iesus en la Prouincia del Piru [...]. En Lima por Francisco del Canto. 